\title{
Hyperbolic systems and propagation on causal manifolds
}

\author{
Pierre Schapira*
}

November 6, 2018

\begin{abstract}
In this paper, which is essentially a survey, we solve the global Cauchy problem on causal manifolds for hyperbolic systems of linear partial differential equations in the framework of hyperfunctions. Besides the classical Cauchy-Kowalevsky theorem, our proofs only use tools and ideas from the microlocal theory of sheaves of [KS90, that is, of purely algebraic and geometric nature. The study of hyperbolic $\mathscr{D}$-modules is only sketched in loc. cit. and the global propagation results are mainly extracted from [DS99].
\end{abstract}

\section{Introduction}

The study of the Cauchy problem in the framework of distributions theory is extremely difficult and there is no characterization of the class of differential operators for which the problem is well posed, although some particular situations are well understood: operators with constant coefficients or operators with simple characteristics (see [Ho83]). To make an history of this subject is out of the scope of this paper and we shall only quote J. Leray [Le53].

If one replaces the sheaf of distributions by the sheaf of Sato's hyperfunctions, the situation drastically simplifies and the Cauchy problem in this setting was solved in [BS73] for a single differential operator and in [KS79] for microfunctions solutions of microdifferential systems. The main difference

*Key words: global propagation, microlocal theory of sheaves, $\mathscr{D}$-modules, hyperbolic systems, hyperfunctions 
between distribution and hyperfunction solutions is that for hyperfunctions the situation is governed by the principal symbol of the operator (or the characteristic variety of the system), contrary to the case of distributions.

However, following [KS90], a new idea has emerged: one can treat hyperfunction solutions of linear partial differential equations (LPDE) from a purely sheaf theoretical point of view, the only analytic tool being the classical Cauchy-Kowalevsky theorem. This idea is developed all along this book and is applied in particular to the study of hyperbolic systems, but this study is performed in a very general setting, with emphasis on the microlocal point of view (see loc. cit. Prop. 11.5.8) and we think it may be useful to give a more direct and elementary approach to hyperbolic systems.

In this paper, we shall show how to solve the Cauchy problem and to treat the propagation of solutions for general systems of LPDE in the framework of hyperfunctions by using the Cauchy-Kowalevsky theorem (and its extension to systems by Kashiwara [Ka70]) and some tools from the microlocal theory of sheaves of [KS90]. Namely, we shall use the microsupport of sheaves, the fact that the microsupport of the complex of holomorphic solutions of a $\mathscr{D}$-module is contained in the characteristic variety of the $\mathscr{D}$-module and a theorem which gives a bound to the microsupport of the restriction to a submanifold of a sheaf.

Hence, after recalling first some elementary facts of differential and symplectic geometry, we shall recall the notion of a system of LPDE on a complex manifold $X$ (that is, a $\mathscr{D}_{X}$-module), the properties of its characteristic variety and the Cauchy-Kowalevsky-Kashiwara theorem (see [Ka70]). Next we introduce the microsupport of sheaves on a real manifold $M$ following [KS90, the natural tool to describe phenomena of propagation. We state the theorem which, given a sheaf $F$ on $M$ and a submanifold $N$ of $M$, gives a bound to the microsupport of the sheaves $\left.F\right|_{N}$ and $\mathrm{R} \Gamma_{N} F$. Then we study LPDE on real manifolds, define the hyperbolic characteristic variety and state the main theorems: one can solve the Cauchy problem for hyperfunction solutions of hyperbolic systems and such solutions propagate in the hyperbolic directions.

Finally, we study global propagation on causal manifolds following [DS99]. We call here a causal manifold a pair $(M, \lambda)$ where $M$ is a smooth connected manifold and $\lambda$ is a closed convex proper cone of the cotangent bundle with non empty interior at each $x \in M$ and such that the order relation $\preceq$ associated with $\lambda$ (by considering oriented curves whose tangents belong to the polar cone to $\lambda$ ) is closed and proper. As an immediate application of our 
results, we find that if $P$ is a differential operator for which the non-zero vectors of $\lambda$ are hyperbolic, then $P$ induces an isomorphism on the space $\Gamma_{A}\left(M ; \mathscr{B}_{M}\right)$ of hyperfunctions on $M$ supported by a closed set $A$ as soon as $A \neq M$ and $A$ is past-like.

As mentioned in the abstract, the study of hyperbolic $\mathscr{D}$-modules is only sketched in KS90 and this is the reason of this paper.

\section{Basic geometry}

In this section, we recall some elementary facts of differentiable and symplectic geometry.

\section{Normal and conormal bundles}

Let $M$ be a real (or complex) manifold. We denote by $\tau: T M \rightarrow M$ its tangent bundle and by $\pi: T^{*} M \rightarrow M$ its cotangent bundle. If $N$ is a submanifold of $M$ we have the exact sequences of vector bundles on $N$ :

$$
\begin{aligned}
& 0 \rightarrow T N \rightarrow N \times{ }_{M} T M \rightarrow T_{N} M \rightarrow 0, \\
& 0 \rightarrow T_{N}^{*} M \rightarrow N \times_{M} T^{*} M \rightarrow T^{*} N \rightarrow 0 .
\end{aligned}
$$

The vector bundle $T_{N} M$ is called the normal bundle to $N$ in $M$ and the vector bundle $T_{N}^{*} M$ is called the conormal bundle to $N$ in $M$. In the sequel, we shall identify $M$ to $T_{M}^{*} M$, the zero-section of $T^{*} M$.

\section{Normal cones}

Let $M$ be a real manifold and let $S, Z$ be two subsets of $M$. The normal cone $C(S, Z)$ is a closed conic subset of $T M$ defined as follows. Choose a local coordinate system in a neighborhood of $x_{0} \in M$. Then

$$
\left\{\begin{array}{l}
v \in T_{x_{0}} M \text { belongs to } C_{x_{0}}(S, Z) \subset T_{x_{0}} M \text { if and only if } \\
\text { there exist sequences }\left\{\left(x_{n}, y_{n}, \lambda_{n}\right)\right\}_{n} \subset S \times Z \times \mathbb{R}_{>0} \text { such that } \\
x_{n} \stackrel{n}{\rightarrow} x_{0}, y_{n} \stackrel{n}{\rightarrow} x_{0}, \lambda_{n}\left(x_{n}-y_{n}\right) \stackrel{n}{\rightarrow} v .
\end{array}\right.
$$

The projection of $C(S, Z)$ on $M$ is the set $\bar{S} \cap \bar{Z}$. If $Z=\{x\}$, one writes $C_{\{x\}}(S)$ instead of $C(S, Z)$. This is a closed cone of $T_{x} M$, the set of limits when $y \in S$ goes to $x$ of half-lines issued at $x$ and passing through $y$. More generally, assume that $N$ is a smooth closed submanifold of $M$. At each 
$x \in N$, the normal cone $C_{x}(Z, N)$ is empty or contains $T_{x} N$. The image of $C(Z, N)$ in the quotient bundle $T_{N} M$ is denoted by $C_{N}(Z)$.

\section{Cotangent bundle}

Let $M$ be a real (or complex) manifold. The manifold $T^{*} M$ is a homogeneous symplectic manifold, that is, it is endowed with a canonical 1-form $\alpha_{M}$, called the Liouville form, such that $\omega_{M}=d \alpha_{M}$ is a symplectic form, that is, a closed non-degenerate 2 -form. In a local coordinate system $x=\left(x_{1}, \ldots, x_{n}\right)$,

$$
\alpha_{M}=\sum_{j=1}^{n} \xi_{j} d x_{j}, \quad \omega_{M}=\sum_{j=1}^{n} d \xi_{j} \wedge d x_{j} .
$$

The 2-form $\omega_{M}$ defines an isomorphism $H: T T^{*} M \simeq T^{*} T^{*} M$ called the Hamiltonian isomorphism.

\section{Normal cones in a cotangent bundle}

Consider the particular case of a smooth Lagrangian submanifold $\Lambda$ of a cotangent bundle $T^{*} M$. The Hamiltonian isomorphism $T T^{*} M \stackrel{\longrightarrow}{\sim} T^{*} T^{*} M$ induces an isomorphism

$$
T_{\Lambda} T^{*} M \stackrel{\sim}{\longrightarrow} T^{*} \Lambda
$$

On the other-hand, consider a vector bundle $\tau: E \rightarrow M$. It gives rise to a morphism of vector bundles over $M, \tau^{\prime}: T E \rightarrow E \times_{M} T M$ which by duality gives the map

$$
\tau_{d}: E \times{ }_{M} T^{*} M \rightarrow T^{*} E .
$$

By restricting to the zero-section of $E$, we get the map:

$$
T^{*} M \hookrightarrow T^{*} E .
$$

Now consider the case of a closed submanifold $N \hookrightarrow M$. Using (2.2) with $E=T_{N}^{*} M$, we get an embedding $T^{*} N \hookrightarrow T^{*} T_{N}^{*} M$ which, using (2.1), gives the embedding

$$
T^{*} N \hookrightarrow T_{T_{N}^{*} M} T^{*} M .
$$


If we choose local coordinates $(x, y)$ on $M$ such that $N=\{y=0\}$ and if one denotes by $(x, y ; \xi d x, \eta d y)$ the associated symplectic coordinates on $T^{*} M$, then $T_{N}^{*} M=\{(x, y ; \xi, \eta) ; y=\xi=0\}$. Denote by $\left(x, v \partial_{y}, w \partial_{\xi}, \eta d y\right)$ the associated coordinates on $T_{T_{N}^{*} M} T^{*} M$. Then the embedding $T^{*} N \hookrightarrow$ $T_{T_{N}^{*} M} T^{*} M$ is described by $(x ; \xi) \mapsto(x, 0 ; \xi, 0)$.

\section{Linear partial differential equations}

References are made to $\mathrm{Ka03}$.

Let $X$ be a complex manifold. One denotes by $\mathscr{D}_{X}$ the sheaf of rings of holomorphic (finite order) differential operators. A system of linear differential equations on $X$ is a left coherent $\mathscr{D}_{X}$-module $\mathscr{M}$. The link with the intuitive notion of a system of linear differential equations is as follows. Locally on $X, \mathscr{M}$ may be represented as the cokernel of a matrix $\cdot P_{0}$ of differential operators acting on the right:

$$
\mathscr{M} \simeq \mathscr{D}_{X}^{N_{0}} / \mathscr{D}_{X}^{N_{1}} \cdot P_{0}
$$

By classical arguments of analytic geometry (Hilbert's syzygies theorem), one shows that $\mathscr{M}$ is locally isomorphic to the cohomology of a bounded complex

$$
\mathscr{M}^{\bullet}:=0 \rightarrow \mathscr{D}_{X}^{N_{r}} \rightarrow \cdots \rightarrow \mathscr{D}_{X}^{N_{1}} \stackrel{\cdot P_{0}}{\longrightarrow} \mathscr{D}_{X}^{N_{0}} \rightarrow 0 .
$$

The complex of holomorphic solutions of $\mathscr{M}$, denoted $\operatorname{Sol}(\mathscr{M})$, (or better in the language of derived categories, $\left.\mathrm{R} \mathscr{H}_{0 m_{\mathscr{D}_{X}}}\left(\mathscr{M}, \mathscr{O}_{X}\right)\right)$, is obtained by applying $\mathscr{H}_{0} m_{\mathscr{D}_{X}}\left(\cdot, \mathscr{O}_{X}\right)$ to $\mathscr{M}^{\bullet}$. Hence

$$
\operatorname{Sol}(\mathscr{M}) \simeq 0 \rightarrow \mathscr{O}_{X}^{N_{0}} \stackrel{P_{0}}{\longrightarrow} \mathscr{O}_{X}^{N_{1}} \rightarrow \cdots \mathscr{O}_{X}^{N_{r}} \rightarrow 0,
$$

where now $P_{0}$. operates on the left.

One defines naturally the characteristic variety of $\mathscr{M}$, denoted char $(\mathscr{M})$, a closed complex analytic subset of $T^{*} X$, conic with respect to the action of $\mathbb{C}^{\times}$on $T^{*} X$. For example, if $\mathscr{M}$ has a single generator $u$ with relation $\mathscr{I} u=0$, where $\mathscr{I}$ is a locally finitely generated left ideal of $\mathscr{D}_{X}$, then

$$
\operatorname{char}(\mathscr{M})=\left\{(z ; \zeta) \in T^{*} X ; \sigma(P)(z ; \zeta)=0 \text { for all } P \in \mathscr{I}\right\},
$$

where $\sigma(P)$ denotes the principal symbol of $P$.

The fundamental result below was obtained in [SKK73]. 
Theorem 3.1. Let $\mathscr{M}$ be a coherent $\mathscr{D}_{X}$-module. Then char $(\mathscr{M})$ is a closed conic complex analytic involutive (i.e., co-isotropic) subset of $T^{*} X$.

The proof of the involutivity is really difficult: it uses microdifferential operators of infinite order and quantized contact transformations. Later, Gabber Ga81] gave a purely algebraic (and much simpler) proof of this result.

\section{Cauchy problem for LPDE}

Let $Y$ be a complex submanifold of the complex manifold $X$ and let $\mathscr{M}$ be a coherent $\mathscr{D}_{X}$-module. One can define the induced $\mathscr{D}_{Y}$-module $\mathscr{M}_{Y}$, but in general it is an object of the derived category $\mathrm{D}^{\mathrm{b}}\left(\mathscr{D}_{Y}\right)$ which is neither concentrated in degree zero nor coherent. Nevertheless, there is a natural morphism

$$
\left.\mathrm{R} \mathscr{H} o m_{\mathscr{D}_{X}}\left(\mathscr{M}, \mathscr{O}_{X}\right)\right|_{Y} \rightarrow \mathrm{R} \mathscr{H} o m_{\mathscr{D}_{Y}}\left(\mathscr{M}_{Y}, \mathscr{O}_{Y}\right) \text {. }
$$

Recall that one says that $Y$ is non-characteristic for $\mathscr{M}$ if

$$
\operatorname{char}(\mathscr{M}) \cap T_{Y}^{*} X \subset T_{X}^{*} X .
$$

With this hypothesis, the induced system $\mathscr{M}_{Y}$ by $\mathscr{M}$ on $Y$ is a coherent $\mathscr{D}_{Y}$-module and one has the Cauchy-Kowalesky-Kashiwara theorem [Ka70]:

Theorem 3.2. Assume $Y$ is non-characteristic for $\mathscr{M}$. Then $\mathscr{M}_{Y}$ is a coherent $\mathscr{D}_{Y}$-module and the morphism (3.2) is an isomorphism.

Example 3.3. Assume $\mathscr{M}=\mathscr{D}_{X} / \mathscr{D}_{X} \cdot P$ for a differential operator $P$ of order $m$ and $Y$ is a hypersurface. In this case, the induced system $\mathscr{M}_{Y}$ is isomorphic to $\mathscr{D}_{Y}^{m}$ and one recovers the classical Cauchy-Kowalesky theorem.

More precisely, choose a local coordinate system $z=\left(z_{0}, z_{1}, \ldots, z_{n}\right)=$ $\left(z_{0}, z^{\prime}\right)$ on $X$ such that $Y=\left\{z_{0}=0\right\}$. Then $Y$ is non-characteristic with respect to $P\left(\right.$ i.e., for the $\mathscr{D}_{X}$-module $\left.\mathscr{D}_{X} / \mathscr{D}_{X} \cdot P\right)$ if and only if $P$ is written as

$$
P\left(z_{0}, z^{\prime} ; \partial_{z_{0}}, \partial_{z^{\prime}}\right)=\sum_{0 \leq j \leq m} a_{j}\left(z_{0}, z^{\prime}, \partial_{z^{\prime}}\right) \partial_{z_{0}}^{j}
$$

where $a_{j}\left(z_{0}, z^{\prime}, \partial_{z^{\prime}}\right)$ is a differential operator not depending on $\partial_{z_{0}}$ of order $\leq m-j$ and $a_{m}\left(z_{0}, z^{\prime}\right)$ (which is a holomorphic function on $X$ ) satisfies: $a_{m}\left(0, z^{\prime}\right) \neq 0$. By the definition of the induced system $\mathscr{M}_{Y}$ we obtain

$$
\mathscr{M}_{Y} \simeq \mathscr{D}_{X} /\left(z_{0} \cdot \mathscr{D}_{X}+\mathscr{D}_{X} \cdot P\right) \text {. }
$$


By the Späth-Weierstrass division theorem for differential operators, any $Q \in \mathscr{D}_{X}$ may be written uniquely in a neighborhood of $Y$ as

$$
Q=R \cdot P+\sum_{j=0}^{m-1} S_{j}\left(z, \partial_{z^{\prime}}\right) \partial_{z_{0}}^{j}
$$

hence, as

$$
Q=z_{0} \cdot Q_{0}+R \cdot P+\sum_{j=0}^{m-1} R_{j}\left(z^{\prime}, \partial_{z^{\prime}}\right) \partial_{z_{0}}^{j}
$$

Therefore $\mathscr{M}_{Y}$ is isomorphic to $\mathscr{D}_{Y}^{m}$. Theorem 3.2 gives:

$$
\left.\mathscr{H}_{0} m_{\mathscr{D}_{X}}\left(\mathscr{M}, \mathscr{O}_{X}\right)\right|_{Y} \simeq \mathscr{O}_{Y}^{m},\left.\quad \mathscr{E}_{x} t_{\mathscr{D}_{X}}^{1}\left(\mathscr{M}, \mathscr{O}_{X}\right)\right|_{Y} \simeq 0
$$

In other words, the morphism which to a holomorphic solution $f$ of the homogeneous equation $P f=0$ associates its $m$-first traces on $Y$ is an isomorphism and one can solve the equation $P f=g$ is a neighborhood of each point of $Y$.

This is exactly the classical Cauchy-Kowalesky theorem. Note that the proof of Kashiwara of the general case is deduced form the classical theorem by purely algebraic arguments.

\section{Microsupport and propagation}

References are made to [KS90].

The idea of microsupport takes its origin in the study of LPDE and particularly in the study of hyperbolic systems. Let $F$ be a sheaf on a real manifold $M$. Roughly speaking, one says that $F$ propagates in the codirection $p=\left(x_{0} ; \xi_{0}\right) \in T^{*} M$ if for any open set $U$ of $M$ such that $x_{0} \in \partial U, \partial U$ is smooth in a neighborhood of $x_{0}$ and $\xi_{0}$ is the exterior normal vector to $U$ at $x_{0}$, any section of $F$ on $U$ extends through $x_{0}$, that is, extends to a bigger open set $U \cup V$ where $V$ is a neighborhood of $x_{0}$.

Example 4.1. (i) Assume $X$ is a complex manifold, $F$ is the sheaf of holomorphic solutions of the equation $P f=0$ where $P$ is a differential operator and $\sigma(P)(p) \neq 0$. Then $F$ propagates in the codirection $p$. This follows easily from the Cauchy-Kowalevsky theorem (see [Ho83, $§ 9.4]$ ). 
(ii) Assume $M=\mathbb{R} \times N$ where $N$ is a Riemannian manifold. Let $P=\partial_{t}^{2}-\Delta$ be the wave equation. (Here, $t$ is the coordinate on $\mathbb{R}$ and $\Delta$ is the Laplace operator on $N$.) Let $F$ be the sheaf of distribution solutions of the equation $P u=0$. Then $F$ propagates at each $p=\left(t_{0}, x_{0} ; \pm 1,0\right)$.

\section{Microsupport}

Let $M$ denote a real manifold of class $C^{\infty}$, let $\mathbf{k}$ be a field, and let $F$ be a bounded complex of sheaves of $\mathbf{k}$-vector spaces on $M$ (more precisely, $F$ is an object of $\mathrm{D}^{\mathrm{b}}\left(\mathbf{k}_{M}\right)$, the bounded derived category of sheaves on $M$ ).

Definition 4.2. Let $F \in \mathrm{D}^{\mathrm{b}}\left(\mathbf{k}_{M}\right)$. The microsupport $\mathrm{SS}(F)$ is the closed $\mathbb{R}^{+}$-conic subset of $T^{*} M$ defined as follows: for an open subset $W \subset T^{*} M$ one has $W \cap \mathrm{SS}(F)=\emptyset$ if and only if for any $x_{0} \in M$ and any real $\mathscr{C}^{1}$ function $\varphi$ on $M$ defined in a neighborhood of $x_{0}$ with $\left(x_{0} ; d \varphi\left(x_{0}\right)\right) \in W$, one has $\left(\mathrm{R} \Gamma_{\left\{x ; \varphi(x) \geq \varphi\left(x_{0}\right)\right\}} F\right)_{x_{0}} \simeq 0$.

In other words, $p \notin \mathrm{SS}(F)$ if the sheaf $F$ has no cohomology supported by "half-spaces" whose conormals are contained in a neighborhood of $p$. Note that the condition $\left(\mathrm{R} \Gamma_{\left\{x ; \varphi(x) \geq \varphi\left(x_{0}\right)\right\}} F\right)_{x_{0}} \simeq 0$ is equivalent to the following: setting $U=\left\{x \in M ; \varphi(x)<\varphi\left(x_{0}\right)\right\}$, one has the isomorphism for all $j \in \mathbb{Z}$

$$
\underset{V \ni x_{0}}{\lim _{V}} H^{j}(U \cup V ; F) \stackrel{\sim}{\longrightarrow} H^{j}(U ; F)
$$

- By its construction, the microsupport is $\mathbb{R}^{+}$-conic, that is, invariant by the action of $\mathbb{R}^{+}$on $T^{*} M$.

- $\operatorname{SS}(F) \cap T_{M}^{*} M=\pi(\mathrm{SS}(F))=\operatorname{Supp}(F)$.

- The microsupport satisfies the triangular inequality: if $F_{1} \rightarrow F_{2} \rightarrow$ $F_{3} \stackrel{+1}{\longrightarrow}$ is a distinguished triangle in $\mathrm{D}^{\mathrm{b}}\left(\mathbf{k}_{M}\right)$, then $\operatorname{SS}\left(F_{i}\right) \subset \operatorname{SS}\left(F_{j}\right) \cup$ $\mathrm{SS}\left(F_{k}\right)$ for all $i, j, k \in\{1,2,3\}$ with $j \neq k$.

In the sequel, for a locally closed subset $A \subset M$, we denote by $\mathbf{k}_{A}$ the sheaf on $M$ which is the constant sheaf with stalk $\mathbf{k}$ on $A$ and is zero on $M \backslash A$.

Example 4.3. (i) If $F$ is a non-zero local system on $M$ and $M$ is connected, then $\mathrm{SS}(F)=T_{M}^{*} M$.

(ii) If $N$ is a closed submanifold of $M$ and $F=\mathbf{k}_{N}$, then $\operatorname{SS}(F)=T_{N}^{*} M$, the conormal bundle to $N$ in $M$. 
(iii) Let $\varphi$ be a $\mathscr{C}^{1}$-function such that $d \varphi(x) \neq 0$ whenever $\varphi(x)=0$. Let $U=\{x \in M ; \varphi(x)>0\}$ and let $Z=\{x \in M ; \varphi(x) \geq 0\}$. Then

$$
\begin{aligned}
& \mathrm{SS}\left(\mathbf{k}_{U}\right)=U \times_{M} T_{M}^{*} M \cup\{(x ; \lambda d \varphi(x)) ; \varphi(x)=0, \lambda \leq 0\}, \\
& \mathrm{SS}\left(\mathbf{k}_{Z}\right)=Z \times_{M} T_{M}^{*} M \cup\{(x ; \lambda d \varphi(x)) ; \varphi(x)=0, \lambda \geq 0\} .
\end{aligned}
$$

For a precise definition of being co-isotropic, we refer to [KS90, Def. 6.5.1].

Theorem 4.4. Let $F \in \mathrm{D}^{\mathrm{b}}\left(\mathbf{k}_{M}\right)$. Then its microsupport $\mathrm{SS}(F)$ is coisotropic.

\section{Microsupport and characteristic variety}

Assume now that $\left(X, \mathscr{O}_{X}\right)$ is a complex manifold and let $\mathscr{M}$ be a coherent $\mathscr{D}_{X}$-module. Recall that one sets for short $\operatorname{Sol}(\mathscr{M}):=\mathrm{R} \mathscr{H}_{o m_{\mathscr{D}_{X}}}\left(\mathscr{M}, \mathscr{O}_{X}\right)$ (see (3.1)).

After identifying $X$ with its real underlying manifold, the link between the microsupport of sheaves and the characteristic variety of coherent $\mathscr{D}$-modules is given by:

Theorem 4.5. (See [KS90, Th. 11.3.3].) Let $\mathscr{M}$ be a coherent $\mathscr{D}_{X}$-module. Then $\operatorname{SS}(\operatorname{Sol}(\mathscr{M}))=\operatorname{char}(\mathscr{M})$.

The inclusion $\operatorname{SS}(\operatorname{Sol}(\mathscr{M})) \subset \operatorname{char}(\mathscr{M})$ is the most useful in practice. By purely algebraic arguments one reduces its proof to the case where $\mathscr{M}=$ $\mathscr{D}_{X} / \mathscr{D}_{X} \cdot P$, in which case this result is due to Zerner [Ze71] who deduced it from the Cauchy-Kowalevsky theorem in its precise form given by Petrovsky and Leray (see also [Ho83, $§ 9.4]$ ). As a corollary of Theorems 4.4 and 4.5, one recovers the fact that the characteristic variety of a coherent $\mathscr{D}_{X}$-module is co-isotropic.

\section{Propagation 1}

Consider a closed submanifold $N$ of $M$ and let $F \in \mathrm{D}^{\mathrm{b}}\left(\mathbf{k}_{M}\right)$. There is a natural morphism

$$
\left.F\right|_{N} \rightarrow \mathrm{R} \Gamma_{N} F \otimes \mathrm{or}_{N / M}[d]
$$

Here, or $_{N / M}$ is the relative orientation sheaf and $d$ is the codimension of $N$. To better understand this morphism, consider the case where $N$ is a 
hypersurface dividing $M$ into two closed half-spaces $M^{+}$and $M^{-}$. Then we have a distinguished triangle

$$
\left.\left.\left.\mathrm{R} \Gamma_{N} F \stackrel{\alpha}{\rightarrow}\left(\mathrm{R} \Gamma_{M^{+}} F\right)\right|_{N} \oplus\left(\mathrm{R} \Gamma_{M^{-}} F\right)\right|_{N} \stackrel{\beta}{\rightarrow} F\right|_{N} \stackrel{+1}{\rightarrow} .
$$

Here $\alpha(u)=(u,-u)$ and $\beta(v, w)=v+w$, but one can also replace the morphism $\alpha$ with $-\alpha$. If one wants morphisms intrinsically defined, then one way is to replace $\mathrm{R} \Gamma_{N} F$ with $\mathrm{R} \Gamma_{N} F \otimes \mathrm{or}_{N / M}$.

The next result will be used when studying the Cauchy problem for hyperfunctions.

Theorem 4.6. (See [KS90, Cor. 5.4.11]) Assume that $\operatorname{SS}(F) \cap T_{N}^{*} M \subset T_{M}^{*} M$. Then the morphism (4.1) is an isomorphism.

When $N$ is a hypersurface, the proof is obvious by (4.2) since it follows from the definition of the microsupport and the hypothesis on $F$ that $\left.\left(\mathrm{R} \Gamma_{M^{ \pm}} F\right)\right|_{N} \simeq 0$. To treat the general case, one uses the Sato's microlocalization functor (see [SKK73] or [KS90]).

\section{Propagation 2}

Let $N \hookrightarrow M$ and $F$ be as above. A natural question is to calculate, or at least to give a bound, to the microsupport of the restriction $\left.F\right|_{N}$ or to $\mathrm{R} \Gamma_{N} F$. The answer is given by

Theorem 4.7. (See [KS90, Cor. 6.4.4].) One has

$$
\begin{aligned}
& \mathrm{SS}\left(\mathrm{R} \Gamma_{N} F\right) \subset T^{*} N \cap C_{T_{N}^{*} M}(\operatorname{SS}(F)), \\
& \mathrm{SS}\left(\left.F\right|_{N}\right) \subset T^{*} N \cap C_{T_{N}^{*} M}(\operatorname{SS}(F)) .
\end{aligned}
$$

Recall that $T^{*} N$ is embedded into $T_{T_{N}^{*} M} T^{*} M$ by (2.3) and the normal cone $C_{T_{N}^{*} M}(\mathrm{SS}(F))$ is a closed subset of $T_{T_{N}^{*} M} T^{*} M$.

\section{$5 \quad$ Hyperbolic systems}

References are made to [KS90].

In this section we denote by $M$ a real analytic manifold of dimension $n$ and by $X$ a complexification of $M$. When necessary, we shall identify the complex manifold $X$ with the real underlying manifold to $X$. 


\section{Hyperfunctions}

We have the sheaves

$$
\mathscr{A}_{M}=\left.\mathscr{O}_{X}\right|_{M}, \quad \mathscr{B}_{M}=H_{M}^{n}\left(\mathscr{O}_{X}\right) \otimes \operatorname{or}_{M}
$$

Here, or $_{M}$ is the orientation sheaf on $M$. The sheaf $\mathscr{A}_{M}$ is the sheaf of real analytic functions on $M$ and the sheaf $\mathscr{B}_{M}$ is the sheaf of Sato's hyperfunctions on $M$ ([Sa59]). It is a flabby sheaf and it contains the sheaf of distributions on $M$ as a subsheaf. Moreover, the cohomology objects $H_{M}^{j}\left(\mathscr{O}_{X}\right)$ are zero for $j \neq n$ and therefore we may better write

$$
\mathscr{B}_{M}=\mathrm{R} \Gamma_{M}\left(\mathscr{O}_{X}\right) \otimes \operatorname{or}_{M}[n]
$$

This will be essential in the proofs of Theorems 5.3 and 5.4 below.

\section{Propagation for hyperbolic systems}

Definition 5.1. Let $\mathscr{M}$ be a coherent left $\mathscr{D}_{X}$-module. We set

$$
\operatorname{hypchar}_{M}(\mathscr{M})=T^{*} M \cap C_{T_{M}^{*} X}(\operatorname{char}(\mathscr{M}))
$$

and call hypchar $\cos _{M}(\mathscr{M})$ the hyperbolic characteristic variety of $\mathscr{M}$ along $M$. A vector $\theta \in T^{*} M$ such that $\theta \notin \operatorname{hypchar}_{M}(\mathscr{M})$ is called hyperbolic with respect to $\mathscr{M}$. In case $\mathscr{M}=\mathscr{D}_{X} / \mathscr{D}_{X} \cdot P$ for a differential operator $P$, one says that $\theta$ is hyperbolic for $P$.

Example 5.2. Assume we have a local coordinate system $z=x+\sqrt{-1} y$ and $M=\{y=0\}$. Denote by $(z ; \zeta)$ the symplectic coordinates on $T^{*} X$ with $\zeta=\xi+\sqrt{-1} \eta$. Let $\left(x_{0} ; \theta_{0}\right) \in T^{*} M$ with $\theta_{0} \neq 0$. Let $P$ be a differential operator with principal symbol $\sigma(P)$. Applying the definition of the normal cone, we find that $\left(x_{0} ; \theta_{0}\right)$ is hyperbolic for $P$ if and only if

$$
\left\{\begin{array}{l}
\text { there exist an open neighborhood } U \text { of } x_{0} \text { in } M \text { and an open conic } \\
\text { neighborhood } \gamma \text { of } \theta_{0} \in \mathbb{R}^{n} \text { such that } \sigma(P)(x ; \theta+\sqrt{-1} \eta) \neq 0 \text { for } \\
\text { all } \eta \in \mathbb{R}^{n}, x \in U \text { and } \theta \in \gamma .
\end{array}\right.
$$

As noticed by M. Kashiwara, it follows from the local Bochner's tube theorem that condition (5.3) will be satisfied as soon as $\sigma(P)\left(x ; \theta_{0}+\sqrt{-1} \eta\right) \neq 0$ for all $\eta \in \mathbb{R}^{n}$ and $x \in U$ (see [BS73]). Hence, one recovers the classical notion of a (weakly) hyperbolic operator (see Le53). 
Theorem 5.3. Let $\mathscr{M}$ be a coherent $\mathscr{D}_{X}$-module. Then

$$
\operatorname{SS}\left(\operatorname{R} \mathscr{H} o_{\mathscr{D}_{X}}\left(\mathscr{M}, \mathscr{B}_{M}\right)\right) \subset \operatorname{hypchar}_{M}(\mathscr{M}) .
$$

The same result holds with $\mathscr{A}_{M}$ instead of $\mathscr{B}_{M}$.

Proof. This follows from Theorem 4.7 and the isomorphisms

$$
\begin{aligned}
& \mathrm{R} \Gamma_{M} \mathrm{R} \mathscr{H} o m_{\mathscr{D}_{X}}\left(\mathscr{M}, \mathscr{O}_{X}\right) \simeq \mathrm{R} \mathscr{H} \operatorname{om}_{\mathscr{D}_{X}}\left(\mathscr{M}, \mathrm{R} \Gamma_{M} \mathscr{O}_{X}\right), \\
& \left.\mathrm{R} \mathscr{H} o m_{\mathscr{D}_{X}}\left(\mathscr{M}, \mathscr{O}_{X}\right)\right|_{M} \simeq \mathrm{R} \mathscr{H} o m_{\mathscr{D}_{X}}\left(\mathscr{M},\left.\mathscr{O}_{X}\right|_{M}\right) .
\end{aligned}
$$

Q.E.D.

\section{Cauchy problem for hyperbolic systems}

We consider the following situation: $M$ is a real analytic manifold of dimension $n, X$ is a complexification of $M, N \hookrightarrow M$ is a real analytic smooth closed submanifold of $M$ of codimension $d$ and $Y \hookrightarrow X$ is a complexification of $N$ in $X$.

Theorem 5.4. Let $M, X, N, Y$ be as above and let $\mathscr{M}$ be a coherent $\mathscr{D}_{X^{-}}$ module. We assume

$$
T_{N}^{*} M \cap \operatorname{hypchar}_{M}(\mathscr{M}) \subset T_{M}^{*} M .
$$

In other words, any non zero vector $\theta \in T_{N}^{*} M$ is hyperbolic for $\mathscr{M}$. Then $Y$ is non characteristic for $\mathscr{M}$ in a neighborhood of $N$ and the isomorphism (3.2) induces the isomorphism

$$
\left.\mathrm{R} \mathscr{H} o m_{\mathscr{D}_{X}}\left(\mathscr{M}, \mathscr{B}_{M}\right)\right|_{N} \sim \operatorname{R} \mathscr{H} o m_{\mathscr{D}_{Y}}\left(\mathscr{M}_{Y}, \mathscr{B}_{N}\right) .
$$

Proof. (i) Since $X$ is a complexification of $M$, there is an isomorphism $M \times_{X} T^{*} X \simeq T_{M}^{*} X \oplus_{M} T^{*} M$. Moreover, there is a natural embedding $T_{M}^{*} X \oplus_{M} T^{*} M \hookrightarrow T_{T_{M}^{*} X} T^{*} X$ (see [KS90, $\left.\S 6.2\right]$ ). Then hypothesis (5.4) implies $T_{N}^{*} M \cap M \times_{X} \operatorname{char}(\mathscr{M}) \subset T_{X}^{*} X$ and since $\operatorname{char}(\mathscr{M})$ is $\mathbb{C}^{\times}$-conic, $N \times_{Y} T_{Y}^{*} X \cap \operatorname{char}(\mathscr{M}) \subset T_{X}^{*} X$. Hence, $Y$ is non characteristic for $\mathscr{M}$.

(ii) We have the chain of isomorphisms

$$
\begin{aligned}
& \left.\mathrm{R} \mathscr{H} o m_{\mathscr{D}_{X}}\left(\mathscr{M}, \mathscr{B}_{M}\right)\right|_{N} \simeq \mathrm{R} \Gamma_{N} \mathrm{R} \mathscr{H} o m_{\mathscr{D}_{X}}\left(\mathscr{M}, \mathscr{B}_{M}\right) \otimes \operatorname{or}_{N / M}[d] \\
& \simeq \mathrm{R} \Gamma_{N} \mathrm{R} \mathscr{H} O m_{\mathscr{D}_{X}}\left(\mathscr{M}, \mathrm{R} \Gamma_{M} \mathscr{O}_{X}\right) \otimes \mathrm{or}_{N}[n+d] \\
& \simeq \mathrm{R} \Gamma_{N} \mathrm{R} \mathscr{H} \operatorname{Om}_{\mathscr{D}_{X}}\left(\mathscr{M}, \mathrm{R} \Gamma_{Y} \mathscr{O}_{X}\right) \otimes \operatorname{or}_{N}[n+d] \\
& \left.\simeq \mathrm{R}_{N} \mathrm{R} \mathscr{H} O m_{\mathscr{D}_{X}}\left(\mathscr{M}, \mathscr{O}_{X}\right)\right|_{Y} \otimes \operatorname{or}_{N}[n-d] \\
& \simeq \mathrm{R} \Gamma_{N} \mathrm{R} \mathscr{H} \operatorname{om}_{\mathscr{O}_{Y}}\left(\mathscr{M}_{Y}, \mathscr{O}_{Y}\right) \otimes \text { or }_{N}[n-d] \\
& \simeq \mathrm{R} \mathscr{H} O m_{\mathscr{D}_{Y}}\left(\mathscr{M}_{Y}, \mathscr{B}_{N}\right) \text {. }
\end{aligned}
$$


Here, the first isomorphism follows from Theorems 5.3 and 4.6, the second uses the definition of the sheaf $\mathscr{B}_{M}$, the third is obvious since $N$ is both contained in $M$ and in $Y$, the fourth follows from Theorems 4.5 and 4.6, the fifth is Theorem 3.2 and the last one uses the definition of the sheaf $\mathscr{B}_{N}$. Q.E.D.

Consider for simplicity the case where $\mathscr{M}=\mathscr{D}_{X} / \mathscr{I}$ where $\mathscr{I}$ is a coherent left ideal of $\mathscr{D}_{X}$. A section $u$ of $\mathscr{H}_{o m_{\mathscr{D}_{X}}}\left(\mathscr{M}, \mathscr{B}_{M}\right)$ is a hyperfunction $u$ such that $Q u=0$ for all $Q \in \mathscr{I}$. It follows that the analytic wave front set of $u$ does not intersect $T_{Y}^{*} X \cap T_{M}^{*} X$ and this implies that the restriction of $u$ (and its derivative) to $N$ is well-defined as a hyperfunction on $N$. One can show that the morphism (5.5) is then obtained using this restriction morphism, similarly as in Theorem 3.2. Since we do not recall the Sato's microlocalization and the notion of wave front set in this paper, we do not explain this point.

Note that Theorem 5.3 and 5.4 were first obtained in [BS73] in case of a single differential operator and in [KS79] in the more general situation of a systems of microdifferential operators acting on microfunctions.

\section{Global propagation on causal manifolds}

There is a vast literature on Lorentzian manifolds (for an exposition, see e.g., the book [BGP07]) but we shall restrict ourselves to recall a global propagation theorem of [DS99] and give applications.

\section{Global propagation for sheaves}

For a manifold $M$ we denote by $q_{1}$ and $q_{2}$ the first and second projection defined on $M \times M$, by $q_{i j}$ the $(i, j)$-th projection defined on $M \times M \times M$ and similarly on $M \times M \times M \times M$. We denote by $\Delta_{M}$ the diagonal of $M \times M$.

A cone $\lambda$ in a vector bundle $E \rightarrow M$ is a subset of $E$ which is invariant by the action of $\mathbb{R}^{+}$on this vector bundle. We denote by $\lambda^{a}$ the opposite

cone to $\lambda$, that is, $\lambda^{a}=-\lambda$ and by $\lambda^{\circ}$ the polar cone to $\lambda$, a closed convex cone of the dual vector bundle

$$
\lambda^{\circ}=\left\{(x, \xi) \in E^{*} ;\langle\xi, v\rangle \geq 0 \text { for all } v \in \lambda\right\} .
$$

In all this section, we assume that $M$ is connected. 
Definition 6.1. Let $Z$ be a closed subset of $M \times M$ and let $A$ be a closed subset of $M$. We say that $A$ is $Z$-proper if $q_{1}$ is proper on $Z \cap q_{2}^{-1}(A)$.

Definition 6.2. (See [DS99, Def. 1.2].) A convex propagator $(Z, \lambda)$ is the data of a closed subset $Z$ of $M \times M$ and a closed convex proper cone $\lambda$ of $T^{*} M$ satisfying

$$
\left\{\begin{array}{l}
\text { (i) } \Delta_{M} \subset Z, \\
\text { (iii) } \operatorname{SS}\left(\mathbf{k}_{Z}\right) \cap\left(T^{*} M \times T_{M}^{*} M \cup T_{M}^{*} M \times T^{*} M\right) \subset T_{M \times M}^{*} M \times M, \\
\text { (iii) } \operatorname{SS}\left(\mathbf{k}_{Z}\right) \subset T^{*} M \times \lambda .
\end{array}\right.
$$

Theorem 6.3. (See [DS99, Cor. 1.4].) Let $(Z, \lambda)$ be a convex propagator and let $A$ be a $Z$-proper closed subset of $M$ with $A \neq M$. Let $F \in \mathrm{D}^{\mathrm{b}}\left(\mathbf{k}_{M}\right)$ and assume that $\mathrm{SS}(F) \cap \lambda^{a} \subset T_{M}^{*} M$ and $\mathrm{SS}\left(\mathbf{k}_{A}\right) \subset \lambda^{a}$. Then $\mathrm{R}_{A}(M ; F) \simeq 0$.

Note that the conclusion of the theorem is equivalent to saying that we have the isomorphism $\mathrm{R} \Gamma(M ; F) \stackrel{\sim}{\sim} \mathrm{R} \Gamma(M \backslash A ; F)$. Roughly speaking, the "sections" of $F$ on $M \backslash A$ extend uniquely to $M$.

\section{Causal manifolds}

In the literature, one often encounters time-orientable Lorentzian manifolds to which one can associate a cone in $T M$ or its polar cone in $T^{*} M$. Here, we only assume that:

$$
\left\{\begin{array}{l}
M \text { is a smooth real connected manifold and we are given a closed } \\
\text { convex proper cone } \lambda \text { in } T^{*} M \text { such that for each } x \in \mathrm{M}, \operatorname{Int}\left(\lambda_{x}\right) \neq \emptyset .
\end{array}\right.
$$

Definition 6.4. A $\lambda$-path is a continuous piecewise $C^{1}$-curve $\gamma:[0,1] \rightarrow M$ such that its derivative $\gamma^{\prime}(t)$ satisfies $\left\langle\gamma^{\prime}(t), v\right\rangle \geq 0$ for all $t \in[0,1]$ and $v \in \lambda$. Here $\gamma^{\prime}(t)$ means as well the right or the left derivative, as soon as it exists (both exist on ]0,1[ and are almost everywhere the same, and $\gamma_{r}^{\prime}(0)$ and $\gamma_{l}^{\prime}(1)$ exist).

To $\lambda$ one associates a preorder on $M$ as follows: $x \preceq y$ if and only if there exists a $\lambda$-path $\gamma$ such that $\gamma(0)=x$ and $\gamma(1)=y$.

For a subset $A$ of $M$, we set:

$$
\begin{aligned}
& A^{\downarrow}=\{x \in M ; \text { there exists } y \in A, x \preceq y\} \\
& A^{\uparrow}=\{x \in M ; \text { there exists } y \in A, y \preceq x\} .
\end{aligned}
$$


We shall assume:

$$
\left\{\begin{array}{l}
\text { the relation } \preceq \text { is closed and proper, that is, } \\
\text { (i) if }\left\{\left(x_{n}, y_{n}\right)\right\}_{n} \text { is a sequence which converges to }(x, y) \text { and } x_{n} \preceq \\
y_{n} \text { for all } n \text {, then } x \preceq y, \\
\text { (ii) for two compact sets } A \text { and } B \text {, the set } B^{\uparrow} \cap A^{\downarrow} \text { is compact. }
\end{array}\right.
$$

Definition 6.5. A pair $(M, \lambda)$ with $\lambda \subset T^{*} M$ satisfying (6.2) and (6.3) will be called here a causal manifold.

Note that if $(M, \lambda)$ is a causal manifold, then so is $\left(M, \lambda^{a}\right)$.

One denotes by $Z_{\lambda}$ the set of $M \times M$ associated with the preorder:

$$
Z_{\lambda}=\{(x, y) \in M \times M ; x \preceq y\} .
$$

Note that giving a relation $\preceq$ satisfying (6.3) is equivalent to giving $Z_{\lambda}$ satisfying:

$$
\left\{\begin{array}{l}
\Delta_{M} \subset Z_{\lambda} \\
q_{13}\left(q_{12}^{-1} Z_{\lambda} \cap q_{12}^{-1} Z_{\lambda}\right) \subset Z_{\lambda} \\
Z_{\lambda} \text { is closed, } \\
q_{13} \text { is proper on } q_{12}^{-1} Z_{\lambda} \cap q_{23}^{-1} Z_{\lambda} .
\end{array}\right.
$$

Note that for a closed subset $A$ of $M$

(i) $A^{\downarrow}=q_{1}\left(Z_{\lambda} \cap q_{2}^{-1} A\right)$ and $A^{\uparrow}=q_{2}\left(Z_{\lambda} \cap q_{1}^{-1} A\right)$,

(ii) if $A$ is compact, the map $q_{1}$ is proper on $Z_{\lambda} \cap q_{2}^{-1} A$ (since $Z_{\lambda}$ is closed),

(iii) if $A$ is compact, then $A^{\downarrow}$ is closed (by (i) and (ii)),

(iv) for two compact sets $A$ and $B$, the set $\left(B^{\uparrow} \times A^{\downarrow}\right) \cap Z_{\lambda}$ is compact (indeed, this set is contained in $\left.\left(B^{\uparrow} \cap A^{\downarrow}\right) \times\left(B^{\uparrow} \cap A^{\downarrow}\right)\right)$,

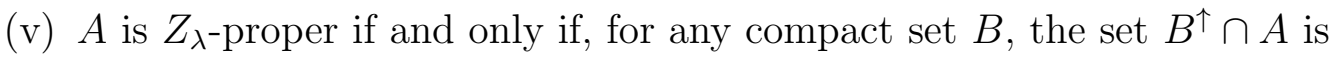
compact. In particular, if $A$ is compact, then $A^{\downarrow}$ is $Z_{\lambda}$-proper.

Proposition 6.6. (See [DS99, Prop. 4.4].) Let $(M, \lambda)$ be a causal manifold. Then

(a) $\left(Z_{\lambda}, \lambda\right)$ is a convex propagator,

(b) if $A$ is a closed subset satisfying $A^{\downarrow}=A$, then $\operatorname{SS}\left(\mathbf{k}_{A}\right) \subset \lambda^{a}$. 
In particular, if $A$ is a closed subset such that $A^{\downarrow}=A^{\uparrow}$, then $\operatorname{SS}\left(\mathbf{k}_{A}\right) \subset$ $T_{M}^{*} M$ and therefore $A=\emptyset$ or $A=M$.

Sketch of proof. To a set $A \subset M$, one associates its strict normal cone $N(A)$ ([KS90, Def. 5.3.6]), an open convex cone of $T M$. In a local coordinate system, $\left(x_{0} ; v_{0}\right) \in N(A)$ if and only if there exists an open cone $\gamma$ containing $v_{0}$ and an open neighborhood $U$ of $x_{0}$ such that

$$
U \cap((A \cap U)+\gamma) \subset A .
$$

One shows that the hypothesis $A^{\downarrow}=A$ implies that $\operatorname{Int}\left(\lambda^{\circ a}\right) \subset N(A)$. Then the proof of (b) follows from the inclusion $\mathrm{SS}\left(\mathbf{k}_{A}\right) \subset N(A)^{\circ}$ ([KS90, Prop. 5.3.8]).

The proof of (a) is similar. $\quad$ Q.E.D.

We can reformulate Theorem 6.3 as follows.

Theorem 6.7. Let $(M, \lambda)$ be a causal manifold. Let $A$ be a closed subset of $M$ such that $A=A^{\downarrow}, A \neq M$ and for any compact subset $B$ of $M$, the set $B^{\uparrow} \cap A$ is compact. Let $F \in \mathrm{D}^{\mathrm{b}}\left(\mathbf{k}_{M}\right)$ and assume that $\operatorname{SS}(F) \cap \lambda^{a} \subset T_{M}^{*} M$. Then $\mathrm{R}_{A}(M ; F) \simeq 0$.

Now let us take for $F$ the complex of hyperfunction solutions of a $\mathscr{D}_{M^{-}}$ module $\mathscr{M}$. We obtain

Corollary 6.8. Let $(M, \lambda)$ and $A$ be as in Theorem6.7. Let $\mathscr{M}$ be a coherent $\mathscr{D}_{X}$-module and assume that $\lambda \cap \operatorname{hypchar}_{M}(\mathscr{M}) \subset T_{M}^{*} M$. In other words, all non-zero vectors of $\lambda$ are hyperbolic for $\mathscr{M}$. Then $\operatorname{RHom}_{\mathscr{D}_{X}}\left(\mathscr{M}, \Gamma_{A} \mathscr{B}_{M}\right) \simeq 0$ or equivalently

$$
\mathrm{R} \Gamma\left(M ; \mathrm{R} \mathscr{H} o m_{\mathscr{D}_{X}}\left(\mathscr{M}, \mathscr{B}_{M}\right)\right) \stackrel{\sim}{\longrightarrow} \Gamma\left(M \backslash A ; \operatorname{R} \mathscr{H} o m_{\mathscr{D}_{X}}\left(\mathscr{M}, \mathscr{B}_{M}\right)\right) .
$$

Example 6.9. Let us particularize to the case of a single differential operator, that is, $\mathscr{M}=\mathscr{D}_{X} / \mathscr{D}_{X} \cdot P$. We find that $P$ induces an isomorphism $\Gamma_{A}\left(M ; \mathscr{B}_{M}\right) \stackrel{\sim}{\longrightarrow} \Gamma_{A}\left(M ; \mathscr{B}_{M}\right)$. In particular

- for any $v \in \Gamma_{A}\left(M ; \mathscr{B}_{M}\right)$, there exists a unique $u \in \Gamma_{A}\left(M ; \mathscr{B}_{M}\right)$ such that $P u=v$,

- any $u \in \Gamma\left(M \backslash A ; \mathscr{B}_{M}\right)$ solution of $P u=0$ extends uniquely all over $M$ as a solution of this equation. 
Corollary 6.10. Let $(M, \lambda)$ be a causal manifold, $N$ a hypersurface which divides $M$ into two closed sets $M^{+}$and $M^{-}$, and let $\mathscr{M}$ be a coherent $\mathscr{D}_{X^{-}}$ module. Assume

(a) $M^{ \pm} \neq M, M^{-}=M^{-\downarrow}, M^{+}=M^{+\uparrow}$ and for any compact subset $B$ of $M$, the sets $B^{\uparrow} \cap M^{-}$and $B^{\downarrow} \cap M^{+}$are compact,

(b) $T_{N}^{*} M \subset \lambda \cup \lambda^{a}$,

(c) $\lambda \cap \operatorname{hypchar}_{M}(\mathscr{M}) \subset T_{M}^{*} M$.

Then the restriction morphism $\mathrm{RHom}_{\mathscr{D}_{X}}\left(\mathscr{M}, \mathscr{B}_{M}\right) \rightarrow \mathrm{RHom}_{\mathscr{D}_{Y}}\left(\left.\mathscr{M}\right|_{Y}, \mathscr{B}_{N}\right)$ is an isomorphism. In other words, the Cauchy problem for hyperfunctions with data on $N$ is globally well-posed.

Remark 6.11. One shall be aware that hypothesis (6.4) may be satisfied on $M$ and not on an open subset of $M$. Following [BGP07, Rem. 3.1.5] consider $M=\mathbb{R} \times \mathbb{R}^{n}$ with linear coordinates $x=\left(x_{0}, x^{\prime}\right)$ and the closed proper cone $\left.\lambda=\left\{x ; \xi_{0}, \xi^{\prime}\right) ; \xi_{0} \geq\left|\xi^{\prime}\right|\right\}$ of $T^{*} M$. It is easy to construct a convex open set $\Omega$ and $x, y \in \Omega$ such that $\{y\}^{\uparrow} \cap\{x\}^{\downarrow} \cap \Omega$ is not compact and to construct a non zero solution $u$ of the equation $P u=0$ on $\Omega$, where $P$ is the wave equation, with support contained in $\{x\}^{\downarrow} \cap \Omega$.

\section{References}

[BGP07] C. Bär, N. Ginoux and F. Pfäffle, Wave Equations on Lorentzian Manifolds and Quantization, ESI Lectures in Mathematics and Physics, (2007)

[BS73] J-M. Bony and P. Schapira, Solutions hyperfonctions du problème de Cauchy, Proceedings Katata 1971, Lecture Notes in Mathematics, Springer-Verlag 287 (1973) pp. 82-98.

[DS99] A. D'Agnolo and P. Schapira, Global propagation on causal manifolds, Asian Math. Journ (1999).

[Ga81] O. Gabber, The integrability of the characteristic variety, Amer. Journ. of Math. 103 (1981) pp 445-468.

[Ho83] L. Hörmander, The analysis of linear partial differential operators I,II Grundlehren der Math. Wiss. 256, 257 Springer-Verlag (1983). 
[Ka70] M. Kashiwara, Algebraic study of systems of partial differential equations, Tokyo 1970 (in Japanese), Mém. Soc. Math. France (translated by A. D’Agnolo and J-P.Schneiders) 63 (1995)

[Ka03] M. Kashiwara, D-modules and Microlocal Calculus, Translations of Mathematical Monographs, 217 American Math. Soc. (2003).

[KS79] M. Kashiwara and P. Schapira, Micro-hyperbolic systems, Acta Math. 142 (1979), pp. 1-55.

[KS90] _ Sheaves on Manifolds, Grundlehren der Math. Wiss. 292 Springer-Verlag (1990).

[Le53] J. Leray, Hyperbolic differential equations, The Institute for Advanced Study, Princeton, N. J., (1953).

[Sa59] M. Sato, Theory of hyperfunctions. I, J. Fac. Sci. Univ. Tokyo. Sect. I 8 (1959), 139-193; II, ibid. 8 (1960), pp. 387-437.

[SKK73] M. Sato, T. Kawai, and M. Kashiwara, Microfunctions and pseudodifferential equations, in Komatsu (ed.), Hyperfunctions and pseudodifferential equations, Proceedings Katata 1971, Lecture Notes in Math. Springer-Verlag 287 (1973) pp. 265-529.

[Ze71] M. Zerner, Domaine d'holomorphie des fonctions vérifiant une équation aux dérivées partielles, C. R. Acad. Sci. 272 (1971) pp. 16461648.

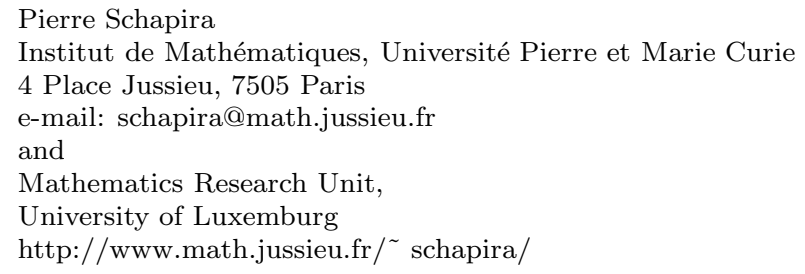

